\author{
SYSTEMS OPTIMIZATION LABORATORY \\ DEPARTMENT OF OPERATIONS RESEARCH \\ STANFORD UNIVERSITY \\ STANFORD, CALIFORNIA 94305-4022
}

DE91 005934

\title{
Two Characterizations of Sufficient Matrices
}

by

\author{
Richard W. Cottle and Sy-Ming Guu
}

TECHNICAL REPORT SOL 90-17

December 1990

\section{DISCLAIMER}

\begin{abstract}
This report was prepared as an account of work sponsored by an agency of the United States Government. Neither the United States Government nor any agency thereof, nor any of their employees, makes any warranty, express or implied, or assumes any legal liability or responsibility for the accuracy, completeness, or usefulness of any information, apparatus, product, or process disclosed, or represents that its use would not infringe privately owned rights. Reference herein to any specific commercial product, process, or service by trade name, trademark, manufacturer, or otherwise does not necessarily constitute or imply its endorsement, recommendation, or favoring by the United States Government or any agency thereof. The views and opinions of authors expressed herein do not necessarily state or reflect those of the United States Government or any agency thereof.
\end{abstract}

Research and reproduction of this report were partially supported by the Department of Energy Grant DE-FG03-87ER25028; National Sc _nce Foundation Grant NSF DMS-8913089 and the Office of Naval Research Grant N00014-89-J-1659.

Any opinions, findings, and conclusions or recommendations expressed in this publication are those of the authors and do NOT necessarily reflect the views of the above sponsors.

Reproduction in whole or in part is permitted for any purposes of the United States Government. This document has been approved for public release and sale; its distribution is unlimited. 


\title{
TWO CHARACTERIZATIONS OF SUFFICIENT MATRICES
}

\author{
by Richard W. COTTLE and Sy-Ming GUU
}

Dedicated to Professor Albert W. Tucker on the occasion of his 85 th birthday.

\begin{abstract}
Two characterizations are given for the class of sufficient matrices defined by Cottle, Pang, and Venkateswaran. The first is a direct translation of the definition into linear programming terms. The second can be thought of as a generalization of a theorem of T.D. Parsons on P-matrices.
\end{abstract}

\section{Introduction}

This note concerns some new classes of matrices that have arisen in connection with the linear complementarity problem [16], [6], namely the row and column sufficient matrices. A matrix $M \in R^{n \times n}$ is said to be column sufficient if for all $x \in R^{n}$

$$
x_{i}(M x)_{i} \leq 0 \quad i=1, \ldots, n \quad \Longrightarrow \quad x_{i}(M x)_{i}=0 \quad i=1, \ldots, n,
$$

and $M$ is said to be row sufficient if $M^{\mathrm{T}}$ is column sufficient. A matrix that is both row and column sufficient is simply called sufficient.

The intrinsic role played by these matrix classes in the theory of linear complementarity problem (LCP) is documented in [7] and will not be repeated here. These matrix classes have algorithmic significance for the LCP as well. It was noted in [7] that nondegenerate linear complementarity problems with row sufficient matrices can be processed by Lemke's method [15]. The same can be said for the principal pivoting method [4] as shown in [2]. Column sufficiency is useful in justifying the least-index degeneracy resolution scheme in connection with the principal pivoting method, and it is conjectured that the same is true for Lemke's method. (See [3].) Row and column sufficient matrices have also recently turned up in papers on interior-point algorithms for the LCP. (See [17], [14].) For all these reasons, it appears that row and column matrices are valuable additions to the literature of the linear complementarity problem.

Up to now, a drawback of these matrices has been the lack of methods for identifying them. The aim of this note is to record two finite tests for sufficiency. Unfortunately, both are combinatorially explosive, hence they cannot be recommended for checking matrices of large order. Nonetheless, it is worthwhile to have some constructive characterizations, especially if they stimulate research leading to more efficient testing methods. 


\section{Basic properties}

The first thing to be said about column (row) sufficient matrices is that they exist. In fact, all $\mathbf{P}$-matrices ${ }^{1}$ are sufficient as are all positive semi-definite matrices (regardless of symmetry). A less familiar matrix class whose elements are all sufficient is $\mathbf{P}_{1}$, the real square matrices having nonnegative principal minors all but one of which are positive. (See [8].) Actually, the column (row) sufficient matrices include the column (row) adequate matrices introduced by Ingleton [12], [13]. (See also Eaves [9].) Hence adequate matrices are sufficient.

All these examples of column (row) sufficient matrices happen to be subclasses of $\mathbf{P}_{0}$ the class of square matrices with nonnegative principal minors. As a matter of fact, a notable property of column sufficient matrices (and hence of row sufficient matrices) is that all their principal minors are nonnegative. (See [7].) This property is far from characterizing these matrices, however. The class $\mathbf{P}_{0}$ is simply too large. The task is to narrow it down appropriately.

Several properties of column (row) sufficient matrices are established in [2]. Among them are the following assertions.

(A1) Every principal rearrangement $P^{\mathrm{T}} M P$ of a column (row) sufficient matrix $M$ is column (row) sufficient.

(A2) If $M$ is column (row) sufficient, then so is $D M D$ for any conformable diagonal matrix $D$.

(A3) Every principal submatrix of a column (row) sufficient matrix is column (row) sufficient.

(A4) A matrix of the form

$$
M=\left(\begin{array}{ll}
a & b \\
0 & 0
\end{array}\right)
$$

with $b \neq 0$ cannot be column sufficient. Its transpose cannot be row sufficient.

(A5) Every principal pivotal transform of a column (row) sufficient matrix is column (row) sufficient.

\section{A test for column sufficiency via linear programming}

The facts that column sufficient matrices have nonnegative principal minors (hence nonnegative diagonal entries) and that all their principal submatrices are column sufficient (see (A3) above) suggests that there ought to be an inductive test for column sufficiency (and likewise row sufficiency). Our aim is here is to exhibit such a test.

Actually, what we test for is violation of the defining condition so that if column sufficiency is verified if the test fails. This approach is akin to the one used in [5] where an inductive test for copositivity was proposed.

\footnotetext{
${ }^{1}$ That is, matrices whose principal minors are all positive. See Gale and Nikaido [11].
} 
Definition. A matrix $M \in R^{n \times n}$ will be called column sufficient of order $r(1 \leq r \leq n)$ if every $r \times r$ principal submatrix of $M$ is column sufficient.

Natural variants of this definition can be used to define row sufficiency of order $r$ and sufficiency of order $r$.

The inductive test for column sufficiency begins with checking the main diagonal for nonnegativity (that is, column sufficiency of order 1 ). The $n \times n$ matrix $M$ is not column sufficient if and only if there exists a vector $x \in R^{n}$ such that

$$
x_{i}(M x)_{i} \leq 0 \quad i=1, \ldots, n \text { and } \sum_{i=1}^{n} x_{i}(M x)_{i}<0 .
$$

Now if $M$ is known to be column sufficient of order $n-1$ where $n-1 \geq 1$, and $x$ satisfies (1), then $x_{i} \neq 0 \quad i=1, \ldots, n$. This says nothing about the signs of the $x_{i}$, however. In principle, all sign $2^{n}$ patterns are eligible, although some can be ruled out on qualitative grounds. For example, (1) has no solution when $x>0$ and $M$ has a semi-positive (nonnegative and nonzero) row. More generally, when the sign pattern of $x$ and that of any row of $M$ imply a positive inner product, the system (1) has no solution. Of course, (1) can be inconsistent for other reasons.

By modifying the matrix, $M$, we may assume that $x>0$. This follows from (A2) by taking $D$ to be the unique diagonal matrix such that $D^{2}=I$ and $D x>0$ for every vector $x$ of a given (all nonzero) sign pattern. Specifically,

$$
x_{i}(M x)_{i}=(D x)_{i}((D M D)(D x))_{i} \quad i=1, \ldots, n .
$$

This being the case, we may assume $x>0$ provided we replace $M$ by $D M D$. When this is done, the modified homogeneous inequality system (1) is equivalent to

$$
\begin{array}{r}
(D M D) x \leq 0 \\
e^{\mathrm{T}}(D M D) x \leq-1 \\
x \geq e
\end{array}
$$

where $e^{\mathrm{T}}=(1, \ldots, 1) \in R^{n}$. One way to check (3) for consistency is to solve the linear program

$$
\begin{aligned}
\text { minimize } & e^{\mathrm{T}}(D M D) x \\
\text { subject to } & -(D M D) x \\
& \geq 0 \\
x & \geq e
\end{aligned}
$$

Note that if $x$ is feasible, so is $\lambda x$ for $\lambda \geq 1$. Thus, if during the solution process the objective function of (LP: $D$ ) turns negative for some feasible (not necessarily optimal) solution, then the computation (relative to (LP: $D)$ ) can be terminated as its objective function is unbounded below.

The prospect of solving $2^{r}$ linear inequality systems or linear programs for each $r \leq n$ is not a happy one, but for small $n$ it is tolerable. 


\section{A generalization of Parsons' Theorem}

Paraphrased, a theorem of Parsons [18] states that a real square matrix $M$ belongs to $\mathbf{P}$ if and only if every principal pivotal transform of $M$ is a $\mathbf{P}$-matrix of order 1. (For a short, seminal paper on principal pivotal transforms of square matrices, see [19].) Our second characterization of sufficient matrices can be viewed as a generalization of Parsons' result.

We begin with a characterization of $2 \times 2$ column sufficient matrices.

Lemma 1. The matrix $M \in R^{2 \times 2}$ is column sufficient if and only if

(i) $M \in \mathbf{P}_{0}$;

(ii) no principal pivotal transform or principal rearrangement of $M$ has the form

$$
\left(\begin{array}{cc}
a & b \\
0 & 0
\end{array}\right) \quad b \neq 0
$$

Proof. The the necessity of (i) and (ii) has already been noted in Section 2. To prove the converse, we may immediately dispense with two extreme cases: $M \in \mathbf{P}$ and $M=0$. Such matrices are column (and row) sufficient. Thus, $M$ must have at least one principal minor that equals 0 . If both diagonal entries are zero, then either $M=0$ or else $M$ has the sign pattern

$$
\left(\begin{array}{ll}
0 & \pm \\
\mp & 0
\end{array}\right),
$$

which is to say that the off-diagonal entries are nonzero and of opposite sign. Matrices of this form are easily shown to be column (and row) sufficient. (See [2].) Suppose $M$ or a principal rearrangement thereof has the sign pattern

$$
\left(\begin{array}{ll}
+ & \pm \\
\mp & 0
\end{array}\right) .
$$

If

$$
\begin{aligned}
& x_{1}\left(m_{11} x_{1}+m_{12} x_{2}\right) \leq 0 \\
& x_{2}\left(m_{21} x_{1}+m_{22} x_{2}\right) \leq 0
\end{aligned}
$$

then

$$
m_{12} x_{1} x_{2} \leq-m_{11} x_{1}^{2} \leq 0 \text { and } m_{21} x_{1} x_{2} \leq 0 .
$$

Since $m_{12} m_{21}<0$, it follows that $x_{1} x_{2}=0$ and then that $x_{1}=0$. This is enough to prove that $M$ is column sufficient. The remaining possibility is that the diagonal entries of $M$ are positive and $M$ is singular. Then $M$ must be column adequate, and such matrices are column sufficient. 
An equivalent way to put Lemma 1 is as follows.

Lemma 2. The matrix $M \in R^{2 \times 2}$ is column sufficient if and only if for every principal pivotal transform $\bar{M}$ of $M$,

(i) $\bar{m}_{i i} \geq 0 \quad i=1,2$;

(ii) for $i=1,2$ if $\bar{m}_{i i}=0$ and $\bar{m}_{i j}=0(j \neq i)$, then $\bar{m}_{j i}=0$.

Proof. Omitted.

Lemma 3. If $M \in R^{n \times n}(n \geq 2)$ and every principal pivotal transiorm of $M$ is column sufficient of order 2 , then $M \in \mathbf{P}_{0}$ and every nonsingular principal submatrix of $M$ can be inverted by a sequence of principal pivots of order 1 or 2 .

Proof. To prove that $M \in \mathbf{P}_{0}$, it is enough to show that $\operatorname{det} M \geq 0$. Indeed, $M$ has a nonnegative diagonal and all principal submatrices of $M$ of order at 2 or more are column sufficient of order 2. This can be used to start an inductive proof. We may assume that $M \neq 0$. Then either $M$ has a positive diagonal entry or it has a positive principal minor of order 2. In either case, a principal pivot of order 1 or 2 is possible. By Schur's determinantal formula (see [1]), the determinant of $M$ equal the determinant of the pivot block (which is positive) times that of the Schur complement of the pivot block. Hence the determinant of $M$ and the determinant of the Schur complement have the same sign. This argument can be repeated until either $M$ is inverted (and hence has a positive determinant) or the Schur complement is a zero matrix (in which case $\operatorname{det} M=0$ ).

For ease of reference in the proof of Theorem 1 below, we state an important characterization of $\mathbf{P}_{0}$-matrices due to Fiedler and Pták [10].

Lemma 4. Let $M \in R^{n \times n}$. Then $M \in \mathbf{P}_{0}$ if and only if for every nonzero vector $x \in R^{n}$ there exists an index $k$ such that $x_{k} \neq 0$ and $x_{k}(M x)_{k} \geq 0$.

Proof. Omitted.

The following simple consequence of Lemma 2 is a characterization of sufficient matrices of order 2.

Lemma 5. The matrix $M \in R^{2 \times 2}$ is (row and column) sufficient if and only if for every principal pivotal transform $\bar{M}$ of $M$,

(i) $\bar{m}_{i i} \geq 0 \quad i=1,2$;

(ii) for $i=1,2$, if $\bar{m}_{i i}=0$, then either $\bar{m}_{i j}=\bar{m}_{j i}=0$ or $\bar{m}_{i j} \bar{m}_{j i}<0$ for $j \neq i$.

Proof. Omitted.

We now wish to characterize sufficient matrices of order 3 or more. 
Theorem 1. Let $M \in R^{n \times n}$ where $n \geq 3$. The following statements are equivalent:

(i) $M$ is sufficient;

(ii) every principal pivotal transform of $M$ is sufficient of order $n-1$.

Proof. The fact that (i) implies (ii) follows from (A5) and (A3). For the converse, we first show that $M$ is column sufficient. Assume $y=M x$ and $x_{i} y_{i} \leq 0$ for $i=1, \ldots, n$ for some vector $x$. In this representation, $y$ is basic (dependent) and $x$ is nonbasic (independent). If any component of $x$ equals zero, it follows from (ii) that $x_{i} y_{i}=0$ for all $i=1, \ldots, n$. Thus, we may assume $x_{i} \neq 0$ for all $i$. If $y_{k}=0$ for some $k$, there are three possibilities: (a) the $k$-th row and column of $M$ are zero in which case (ii) implies $x_{i} y_{i}=0$ for all $i$; (b) it is possible to pivot on $m_{k k}>0$ in which case $y_{k}=0$ plays the role of $x_{k}$ above; (c) it is possible to carry out a block pivot of order 2 in which $y_{k}=0$ again plays the role of a nonbasic variable whose value is zero, and the preceding argument applies. The remaining situation is the one where $x_{k} y_{k}<0$ for all $k$, and we need to show that this cannot occur. We do this by proving that $M \in \mathbf{P}_{0}$ and invoking Lemma 4. Clearly $M$ is $\mathbf{P}_{0}$ of order $n-1$, the proof will be complete once it is shown that $\operatorname{det} M \geq 0$. Now if $M$ has a positive diagonal entry, it can be used as a pivot element to generate a principal pivotal transform of $M$. The corresponding Schur complement is a sufficient matrix of order $n-1$ and as such has a nonnegative determinant. But the determinant of the Schur complement is $\operatorname{det} M$ divided by the pivot element. Hence $\operatorname{det} M$ is nonnegative. If the diagonal of $M$ is zero and $M$ has a row of zeros, then its determinant is zero. If $M$ has a zero diagonal but no row of zeros, then it has a nonsingular 2 by 2 principal submatrix with a positive determinant. When used as a pivot block, this submatrix gives rise to a Schur complement which by hypothesis must be a sufficient matrix of order $n-2$ and accordingly must have a nonnegative determinant. Once again, the Schur determinantal formula implies that $\operatorname{det} M \geq 0$. This shows that $M$ must be a $\mathbf{P}_{0}$-matrix.

As an easily proved consequence, we obtain the aforementioned generalization of Parsons' theorem; it runs as follows.

Theorem 2. Let $M \in R^{n \times n}$ where $n \geq 2$. The following statements are equivalent:

(i) $M$ is sufficient;

(ii) every principal pivotal transform of $M$ is sufficient of order 2 .

Proof. For $n=2$, the theorem is trivial; for $n \geq 3$, the result follows by repeated application of Theorem 1. 


\section{References}

[1] R.W. Cottle, "Manifestations of the Schur complement", Linear Algebra and Its Applications 8 (1974) 189-211.

[2] R.W. Cottle, "The principal pivoting method revisited", Mathematical Programming, Series B 48 (1990) 369-385.

[3] R.W. Cottle and Y.Y. Chang, "Least-index resolution of degeneracy in linear complementarity problems with sufficient matrices", Technical report SOL 90-9, Systems Optimization Laboratory, Department of Operations Research, Stanford University (Stanford, California, 1990). To appear in SIAM Journal on Matrix Analysis and Applications.

[4] R.W. Cottle and G.B. Dantzig, "Complementary pivot theory of mathematical programming", Linear Algebra and Its Applications 1 (1968) 103-125.

[5] R.W. Cottle, G.J. Habetler and C.E. Lemke, "On classes of copositive matrices", Linear Algebra and Its Applications 3 (1970) 295-310.

[6] R.W. Cottle, J.S. Pang, and R.E. Stone, The Linear Complementarity Problem, forthcoming.

[7] R.W. Cottle, J.S. Pang, and V. Venkateswaran, "Sufficient matrices and the linear complementarity problem", Linear Algebra and Its Applications 114/115 (1989) 231-249.

[8] R.W. Cottle and R.E. Stone, "On the uniqueness of solutions to linear complementarity problems", Mathematical Programming 27 (1983) 191-213.

[9] B.C. Eaves, "The linear complementarity proilem", Management Science 17 (1971) 612-634.

[10] M. Fiedler and V. Pták, "Some generalizations of positive definiteness and monotonicity", Numerische Mathematik 9 (1966) 163-172.

[11] D. Gale and H. Nikaido, "The Jacobian matrix and the global univalence of mappings", Mathematische Annalen 159 (1965) 81-93.

[12] A.W. Ingleton, "A problem in linear inequalities", Proceedings of the London Mathematical Socieiy 16 (1.966) 519-536.

[13] A.W. Ingleton, "The linear complementarity problem", Journal of the London Mathematical Society 2 (1970) 330-336.

[14] M. Kojima, N. Megiddo, T. Noma and A. Yoshise, "A unified approach to interior point algorithms for linear complementarity pioblems", forthcoming.

[15] C.E. Lemke, "Bimatrix equilibrium points and mathematical programming", Management Science 11 (1965) 681-689. 
[16] K.G. Murty, Linear Complementarity, Linear and Nonlinear Programming, Heldermann Verlag, Berlin, 1988.

[17] J.S. Pang, "Iterative descent $a_{c}{ }_{c}{ }^{\circ}$ orithms for a row sufficient linear complementarity problem", manuscript (second revision), Department of Mathematical Sciences, Johns Hopkins University, January 1990.

[18] T.D. Parsons, "Applications of principal pivoting", in (H.W. Kuhn, ed.) Proceedings of the Princeton Symposium on Mathematical Programmi.ng, Princeton University Press, Princeton, 1970, pp. 567-581.

[19] A.W. Tucker, "Principal pivota! transforms of square matrices", SIAM Review 5 (1963) 305. 


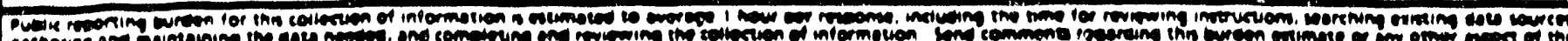

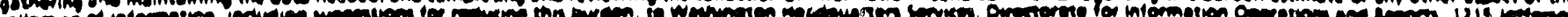

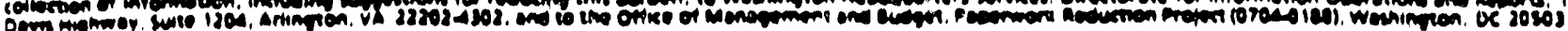

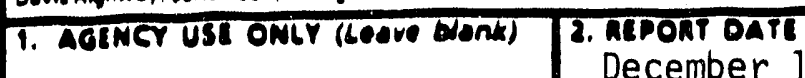
December 1991

3. REPORT TrR AND DATES COVITLO

\section{TITLE AND SUDTITLE}

Tectinical Report

Two Characterizations of Sufficient Matrices

\section{AUTHOR(S)}

Richard W. Cottle and Sy-Ming Guu

\section{PERFORMING ORGANIZATION NAME(S) AND ADDACSSIES)}

Department of Operations Research - SOL

Stanford University

Stanford, CA 94305-4022
Office of Naval Research - Department of the Navy

800 W. Quincy Street

Arlington, VA $222 i 7$

Office of Energy Research

U.S. Department of Energy

Washington, DC 20585

\section{PUMDINO NUMURAS}

DE-FG03-87ER25028

N00014-89-J-1659
12. OISTRIBUTION AVALAOILITY STATEMENT

UNL IMITED
0. PEthFonming organization REPORT NUMERA

$1111 \mathrm{MA}$

10. SPONSORING/MONITORINC AGENCY REPOAT NUMAER

SOL $90-17$

13. ACSTRMCT (Maximum 200 woras)

Two characterizations are given for the class of sufficient matrices defined by Cottle, Pang and Venkateswaran. The first is a direct translation of the definition into linear programming terms. The second can be thought of as a generalization of a theorem of T.D. Parsons on P-matrices.

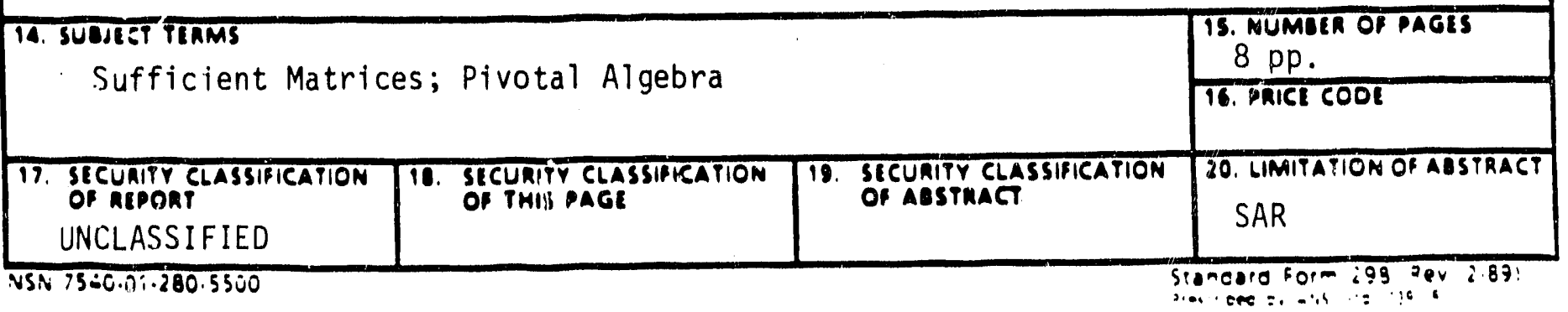



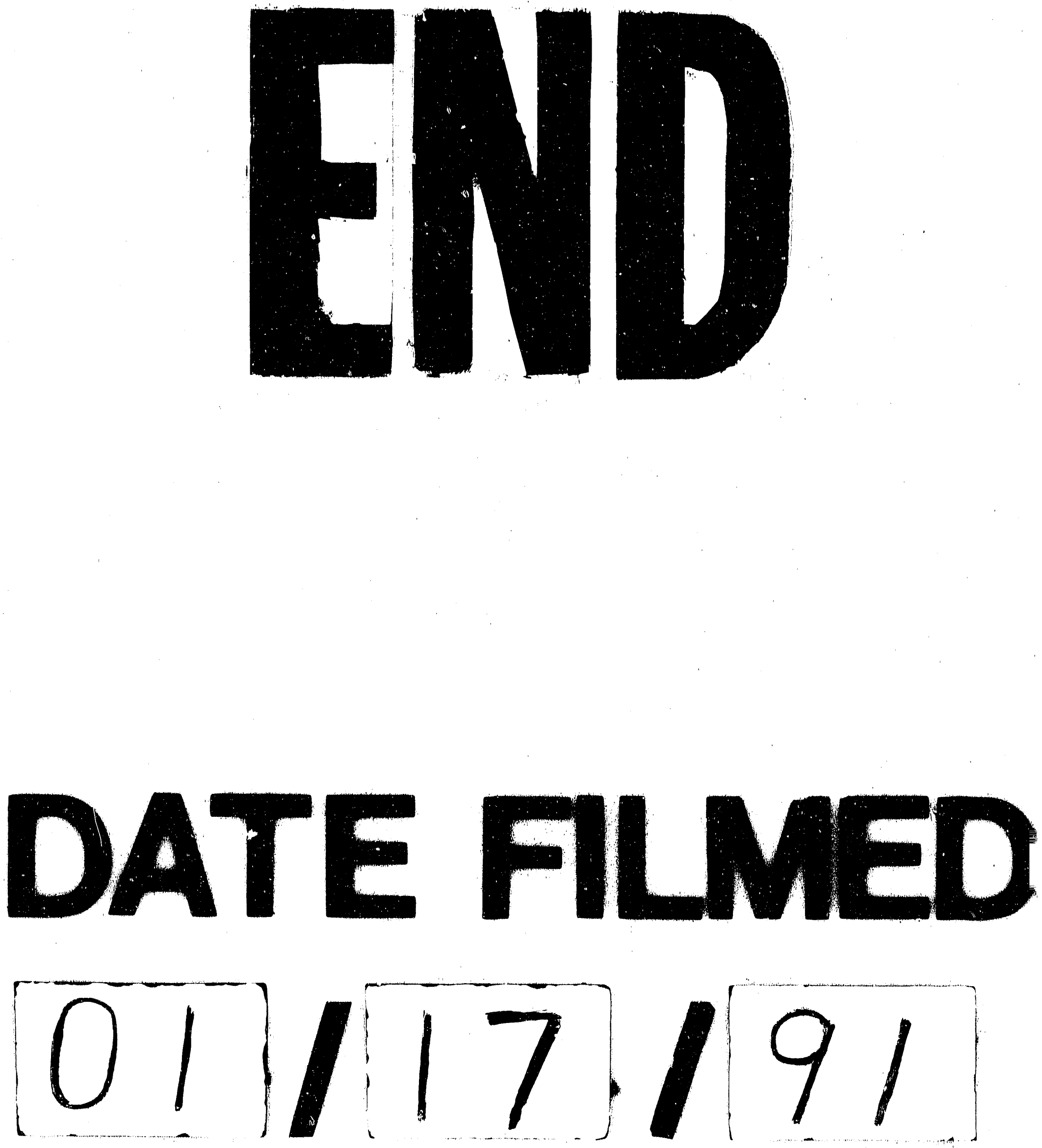
